\title{
Método TLS Truncado para o Problema de Espectroscopia de Ressonância Magnética
}

\author{
Jonathan Ruiz Quiroz \\ UFSC - Departamento de Matemática \\ Campus Trindade \\ 88040-900, Florianópolis, SC \\ E-mail: Jonathan17r@gmail.com. \\ Fermín S. V. Bazán \\ UFSC - Departamento de Matemática \\ Campus Trindade \\ 88040-900, Florianópolis, SC \\ E-mail: fermin@mtm.ufsc.br
}

Resumo: No problema de quadrados mínimos $\min \|A x-b\|$, com $A \in \mathbb{R}^{m \times n}, m \geq n$, e $b \in \mathbb{R}^{m}$, é assumido frequentemente que a matriz $A$ é exata e o vetor $b$ é contaminado por erros. Esta hipótese não é sempre realista, pois existen problemas onde a matriz A também é sujeita a erros. Uma maneira de lidar com problemas desta natureza é através de método de Quadrados Mínimos Totais (TLS). Neste trabalho usamos o método TLS para resolver o problema de espectroscopia em ressonância magnética e apresentamos uma comparação de resultados obtidos com o método dos Quadrados Mínimos (LS).

Palavras-chave: SVD, Quadrados mínimos totais, Espectroscopia em Ressonância Magnética

\section{Introdução}

Problemas que envolvem matrizes contaminadas por ruídos aparecem em áreas das ciências, como biologia, física e engenharia. Nesses casos a técnica de quadrados mínimos pode produzir resultados insatisfatórios. Golub e Van Loan [3] e Van Huffel e Vandewalle [7] desenvolveram uma análise do problema para o caso em que ambos a matriz $A$ e o vetor $b$ contém incertezas e introduziram o método de Quadrados Mínimos Totais. Para entender a filosofia da técnica TLS, é conveniente introduzir a solução do problema de Quadrados Mínimos (LS) dada por

$$
x_{L S}=\underset{x \in \mathbb{R}^{n}}{\operatorname{argmin}}\|A x-b\| .
$$

A solução $x_{L S}$ pode ser determinada a través da Descomposição em Valores Singulares (SVD) da matriz $A[4]: A=U \Sigma V^{T}$, onde $U=\left[u_{1}, \ldots, u_{m}\right] \in \mathbb{R}^{m \times m}, V=\left[v_{1}, \ldots, v_{n}\right] \in \mathbb{R}^{n \times n}$ são matrizes ortogonais e $\Sigma=\operatorname{diag}\left(\sigma_{1}, \ldots, \sigma_{n}\right)$, onde os números $\sigma_{i}$ são os valores singulares de $A$ e são ordenados de modo que $\sigma_{1} \geq \sigma_{2} \geq \ldots \geq \sigma_{n} \geq 0$. Se o vetor de dados é da forma $b=b_{\text {exato }}+e$, com $b_{\text {exato }}$ o vetor sem pertubações e $e$ o vetor de incertezas, e $\operatorname{posto}(A)=n$, usando a SVD obtemos

$$
x_{L S}=\sum_{i=1}^{n} \frac{u_{i}^{T} b}{\sigma_{i}} v_{i} .
$$

Devido à divisão por pequenos valores singulares, a solução $x_{L S}$ pode ser dominada por componentes associadas ao vetor de erros $e$. Portanto, é necessário estabilizar a solução. Uma maneira de amenizar o efeito da influência do erro na solução é truncando a soma em (1.1) para $s \leq n$ termos:

$$
x_{L S}^{s}=\sum_{i=1}^{s} \frac{u_{i}^{T} b}{\sigma_{i}} v_{i}
$$


onde $s$, chamado índice de truncamento, é escolhido de modo que exista um balanço apropriado entre a qualidade da informação do problema que é capturada e a quantidade de erro que é incluída na solução. Este método é conhecido como o método da SVD Truncada (TSVD) [5].

$\mathrm{O}$ desenvolvimento da técnica TLS foi motivada por problemas lineares $A x \approx b, m>n$, onde ambos a matriz $A$ e o vetor $b$ são contaminados por erros. Para amenizar a presença dos erros a técnica TLS considera a matriz aumentada $\left[\begin{array}{ll}A & b\end{array}\right]$ e procura por algum vetor não nulo $x$ tal que

$$
\left[\begin{array}{ll}
A & b
\end{array}\right]\left[\begin{array}{c}
x \\
-1
\end{array}\right]=0 \text {. }
$$

Obviamente, o problema acima tem solução $x \neq 0$ só quando o espaço nulo da matriz aumentada é distinto do espaço trivial, o que não é satisfeito em geral quando ambos $A$ e $b$ são contaminados por erros. Para contornar esta incompatibilidade a técnica TLS considera a SVD da matriz ampliada

$$
\left[\begin{array}{ll}
A & b
\end{array}\right]=\bar{U} \bar{\Sigma} \bar{V}^{\mathbf{T}}
$$

e particiona as matrizes $\bar{V}$ e $\bar{\Sigma}$ como:

$$
\bar{V}=\left[\bar{v}_{1}, \ldots, \bar{v}_{n+1}\right]=\left[\begin{array}{cc}
V_{11} & \bar{v}_{12} \\
\bar{v}_{21}^{T} & \bar{v}_{22}
\end{array}\right], \quad \bar{\Sigma}=\left[\begin{array}{cc}
\bar{\Sigma}_{1} & 0 \\
0 & \bar{\sigma}_{n+1} \\
0 & 0
\end{array}\right],
$$

em que $\bar{V}_{11}, \bar{\Sigma}_{1} \in \mathbb{R}^{n \times n}$ e $\bar{v}_{12}, \bar{v}_{21} \in \mathbb{R}^{n}$. Se $\bar{\sigma}_{n+1} \neq 0$, então posto( $\left.\left[\begin{array}{ll}A & b\end{array}\right]\right)=n+1$, e o espaço nulo da matriz ampliada é trivial, logo o conjunto de equações (1.3) não é compatível. Para obter uma solução, o posto da matriz aumentada, $\left[\begin{array}{ll}A & b\end{array}\right]$ deve ser reduzido de $n+1$ par $n$ e isto pode ser feito aproximando $\left[\begin{array}{ll}A & b\end{array}\right]$ por uma matriz $\left[\begin{array}{ll}A_{n} & b_{n}\end{array}\right]$ de posto $n$. O método TLS procura por um matriz $\tilde{A}$ e um vetor $\tilde{b}$ tais que

$$
\min _{\substack{\tilde{A} \\
\tilde{b}] \in \mathbb{R}^{m \times(n+1)} \\
\tilde{A} x=\tilde{b}}}\left\|\left[\begin{array}{ll}
A & b
\end{array}\right]-\left[\begin{array}{ll}
\tilde{A} & \tilde{b}
\end{array}\right]\right\|_{F}^{2},
$$

onde a $\|\cdot\|_{F}$ denota a norma Frobenius de uma matriz. A solucão do problema de minimização (1.6) é dado pelo teorema de Eckart-Young-Mirsky [7]:

$$
\left[\begin{array}{ll}
A_{n} & b_{n}
\end{array}\right]=\sum_{i=1}^{n} \bar{\sigma}_{i} \bar{u}_{i} \bar{v}_{i}^{T}
$$

que é a matriz de posto $n$ mais próxima de $\left[\begin{array}{ll}A & b\end{array}\right]$ no sentido da norma Frobenius, satisfazendo

$$
\left[\begin{array}{ll}
A & b
\end{array}\right]-\left[\begin{array}{ll}
A_{n} & b_{n}
\end{array}\right]=\bar{\sigma}_{n+1} \bar{u}_{n+1} \bar{v}_{n+1}^{T}, \quad \text { com } \quad\left\|\left[\begin{array}{ll}
A & b
\end{array}\right]-\left[\begin{array}{ll}
A_{n} & b_{n}
\end{array}\right]\right\|_{F}=\bar{\sigma}_{n+1} .
$$

O sistema homogêneo (1.3) é então substituido pelo sistema homogêneo com matriz de posto $n$, $\left[\begin{array}{ll}A_{n} & b_{n}\end{array}\right]$, e usando o fato de que $v_{n+1} \in \mathcal{N}\left(\left[\begin{array}{ll}A_{n} & b_{n}\end{array}\right]\right)$, se $\left[\bar{v}_{n+1}\right]_{n+1} \neq 0$, temos que

$$
\left[\begin{array}{c}
x \\
-1
\end{array}\right]=-\frac{1}{\left[\bar{v}_{n+1}\right]_{n+1}} \bar{v}_{n+1} \text {. }
$$

Portanto, usando (1.5) temos que a solução TLS pode ser expressa como:

$$
x_{T L S}=-\frac{\bar{v}_{12}}{\bar{v}_{22}} .
$$

Note que se $\bar{\sigma}_{n+1}=0$, então $\left[\begin{array}{ll}A & b\end{array}\right]$ tem posto $n$. Neste caso o sistema é compatível e não precisamos aproximar a matriz ampliada. Também, se $\bar{\sigma}_{n+1}$ é um valor singular simple, temos que $\mathcal{N}\left(\left[\begin{array}{ll}A_{n} & b_{n}\end{array}\right]\right)=\operatorname{span}\left\{\bar{v}_{n+1}\right\}$ e a solução TLS é única.

Neste trabalho, apresentamos um estudo comparativo das técnicas LS e TLS aplicadas ao problema de espectroscopia de ressonância magnética, para o caso em que a matriz $\left[\begin{array}{ll}A & b\end{array}\right]$ tem dados inexatos. 


\section{Método de Quadrados Mínimos Totais Truncado}

O método TLS truncado é similar ao método TSVD visto acima. Quando os menores valores singulares de $\left[\begin{array}{ll}A & b\end{array}\right]$ são muito pequenos, truncamos a partir de un índice $k$. Para determinar o índice $k$ adequado é necessário usar algum método que permita estabelecer um bom balanço entre a qualidade da solução e o tamanho do resíduo. Pelo teorema de Eckart-Young-Mirsky a matriz

$$
\left[\begin{array}{ll}
A_{k} & b_{k}
\end{array}\right]=\sum_{i=1}^{k} \bar{\sigma}_{i} \bar{u}_{i} \bar{v}_{i}^{T}
$$

é a melhor aproximação de posto $k$ da matriz $\left[\begin{array}{ll}A & b\end{array}\right]$ no sentido da norma Frobenius. O espaço nulo da matriz aproximação é dado por:

$$
\mathcal{N}\left(\left[\begin{array}{ll}
A_{k} & b_{k}
\end{array}\right]\right)=\operatorname{span}\left\{\bar{v}_{k+1}, \ldots \bar{v}_{n+1}\right\},
$$

que fornece a partição

$$
\bar{V}=\left[\bar{v}_{1}, \ldots, \bar{v}_{n+1}\right]=\left[\begin{array}{ll}
\bar{V}_{11} & \bar{V}_{12} \\
\bar{v}_{21}^{T} & \bar{v}_{22}^{T}
\end{array}\right]
$$

onde $\bar{V}_{11} \in \mathbb{R}^{n \times k}, \bar{V}_{12} \in \mathbb{R}^{n \times(n-k+1)}$, e

$$
\begin{aligned}
& \bar{v}_{21}=\left[\left[\bar{v}_{1}\right]_{n+1}, \ldots,\left[\bar{v}_{k}\right]_{n+1}\right]^{T} \in \mathbb{R}^{k}, \\
& \bar{v}_{22}=\left[\left[\bar{v}_{k+1}\right]_{n+1}, \ldots,\left[\bar{v}_{n+1}\right]_{n+1}\right]^{T} \in \mathbb{R}^{n-k+1}
\end{aligned}
$$

Procuramos por uma solução no núcleo de $\left[\begin{array}{ll}A_{k} & b_{k}\end{array}\right]$. Usando o método em [1] se $\bar{v}_{22} \neq 0$ temos que a solucão TLS é

$$
x_{T L S}^{k}=-\frac{1}{\left\|\bar{v}_{22}\right\|^{2}} \bar{V}_{12} \bar{v}_{22} .
$$

í imediato provar que a norma da solução TLS truncada satisfaz $\left\|x_{T L S}^{k}\right\|^{2}=\frac{1}{\left\|\bar{v}_{22}\right\|^{2}}-1$ e que a norma do resíduo é $\left\|R_{k}\right\|_{F}^{2}=\left\|\left[\begin{array}{ll}A & b\end{array}\right]-\left[\begin{array}{ll}A_{k} & b_{k}\end{array}\right]\right\|_{F}^{2}=\bar{\sigma}_{k+1}^{2}+\ldots+\bar{\sigma}_{n+1}^{2}$. Isso mostra que a norma da solução $\left\|x_{T L S}^{k}\right\|$ cresce como uma função de $k$, e que a norma do residuo $\left\|R_{k}\right\|_{F}$ decresce. Este comportamento sugere usar o Principio da Discrepancia ou o Método da Curva L [1] para encontrar o índice de truncamento $k$ adequado.

\section{Resultados Numéricos Preliminares}

Nesta seção apresentamos um estudo comparativo da eficiência dos métodos LS e TLS quando aplicados a um problema da área de espectroscopia de ressonância magnética (MRS). Neste caso a matriz de dados $A$ tem estrutura Hankel, i.e., as entradas são definidas como $a_{i, j}=h_{i+j-1}$

$$
A=\left[\begin{array}{cccc}
h_{1} & h_{2} & \ldots & h_{n} \\
h_{2} & h_{3} & \ldots & h_{n+1} \\
\vdots & \vdots & \ddots & \vdots \\
h_{n} & h_{n+1} & \ldots & h_{n+m}
\end{array}\right]
$$

e $b=\left[h_{0}, \ldots, h_{n-1}\right]^{T}$, onde $n+m \leq q$, e $h_{k}$ é um sinal modelado por

$$
h_{k}=\sum_{j=1}^{p} c_{j} e^{\iota \phi j} e^{\left(\alpha_{j}+\iota \omega_{j}\right) k \Delta t}, \quad \iota=\sqrt{-1}, \quad k=0,1, \ldots, q .
$$

O problema consiste em estimar os parâmetros $\alpha_{j}, \beta_{j}, \phi_{j}$, e $c_{j}$, a partir de medidas experimentais do sinal $h_{k}$. Se $h_{k}$ é livre de erros, é conhecido que posto $(A)=p$ e a solução de norma mínima do 
problema min $\|A x-b\|$ pode ser usada para estimar as constantes de interesse a través de técnicas de predição linear [2]. A principal dificuldade do problema é que, como o sinal experimental é da forma $\widetilde{h}_{k}=h_{k}^{\text {exato }}+e_{k}$, então a matriz é da forma $\widetilde{A}=A+E$, com posto completo e vetor de dados é $\widetilde{b}=b^{\text {exato }}+e$. Maiores informações podem ser encontradas na referência [2].

Para nosso exemplo consideramos os valores da tabela 1, com $p=11, m=n=256, q=600$, $\Delta t=0.000333$ e $\phi_{j}=\xi_{j} \pi / 180$.

Tabela 1: Valores exatos para o sinal MRS

\begin{tabular}{ccccc}
\hline$j$ & $c_{j}$ & $\xi_{j}$ (graus) & $\alpha_{j}$ & $\omega_{j} / 2 \pi(\mathrm{Hz})$ \\
\hline 1 & 75 & 135 & 50 & -86 \\
2 & 150 & 135 & 50 & -70 \\
3 & 75 & 135 & 50 & -54 \\
4 & 150 & 135 & 50 & 152 \\
5 & 150 & 135 & 50 & 168 \\
6 & 150 & 135 & 50 & 292 \\
7 & 150 & 135 & 50 & 308 \\
8 & 150 & 135 & 25 & 360 \\
9 & 1400 & 135 & 285 & 440 \\
10 & 60 & 135 & 25 & 490 \\
11 & 500 & 135 & 200 & 530 \\
\hline
\end{tabular}

Consideramos matriz perturbada $\widetilde{A}$, sendo $\widetilde{A}=A+\sigma \mathcal{E}$, onde $\mathcal{E}$ é uma matriz de ruido gaussiano, $\sigma$ é o desvio padrão nas partes real e imaginária. Note que a matriz exata $A$ tem posto 11.

A figure abaixo mostra a parte real do sinal usado no experimento.

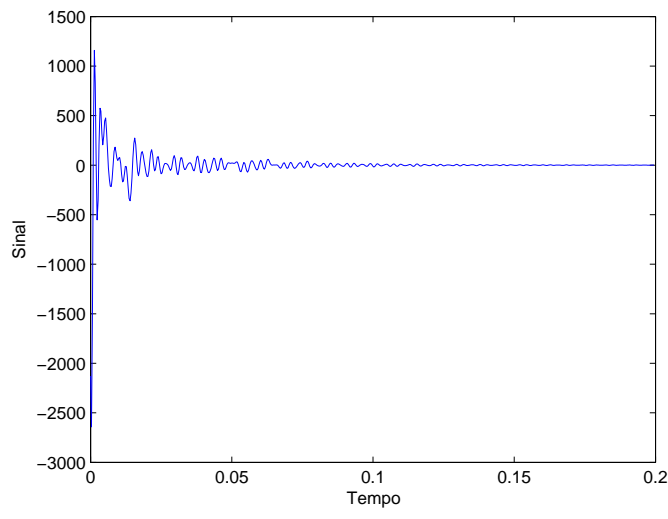

Figura 1: Sinal pura

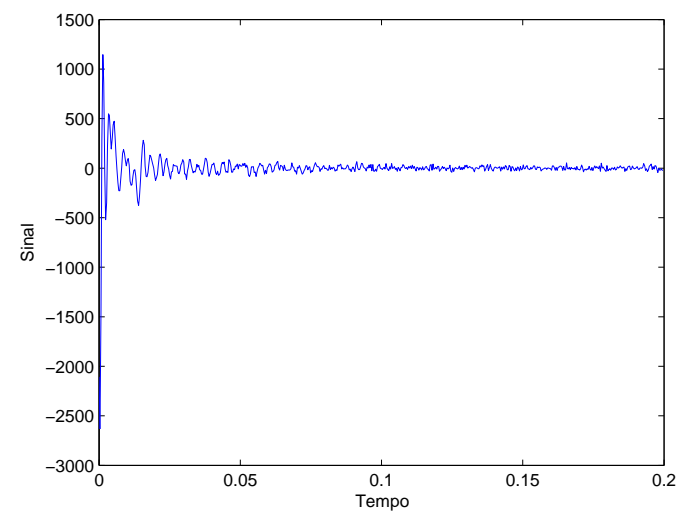

Figura 2: Sinal Perturbada

Apresentamos resultados obtidos com a técnica LS truncada e TLS truncada usando em ambos os casos o índice de truncamento $k=11$, considerando vários valores do desvio padrão.

A qualidade das soluções $x_{L S}, x_{T L S}$ em termos de erro relativo e resíduos relativos com respeito à solução original $x_{0}$ são mostrados na tabela 2 e ilustrados graficamente na figura 3 . 
Tabela 2: Erro relativo e Resíduo relativo

\begin{tabular}{ccccc}
\hline$\sigma$ & $\left\|x_{L S}-x_{0}\right\| /\left\|x_{0}\right\|$ & $\left\|x_{T L S}-x_{0}\right\| /\left\|x_{0}\right\|$ & $\left\|A x_{L S}-b\right\| /\|b\|$ & $\left\|A x_{T L S}-b\right\| /\|b\|$ \\
\hline 2 & 0.0162765 & 0.0163174 & 0.0020342 & 0.0020571 \\
4 & 0.0326695 & 0.0327974 & 0.0039712 & 0.0040338 \\
6 & 0.0492881 & 0.0495050 & 0.0058708 & 0.0059408 \\
8 & 0.0662289 & 0.0665041 & 0.0078041 & 0.0077932 \\
10 & 0.0835820 & 0.0838643 & 0.0098491 & 0.0096115 \\
12 & 0.1014382 & 0.1016722 & 0.0120859 & 0.0114216 \\
14 & 0.1198999 & 0.1200591 & 0.0145900 & 0.0132551 \\
16 & 0.1391069 & 0.1392916 & 0.0174291 & 0.0151501 \\
18 & 0.1593645 & 0.1603396 & 0.0206730 & 0.0171669 \\
\hline
\end{tabular}

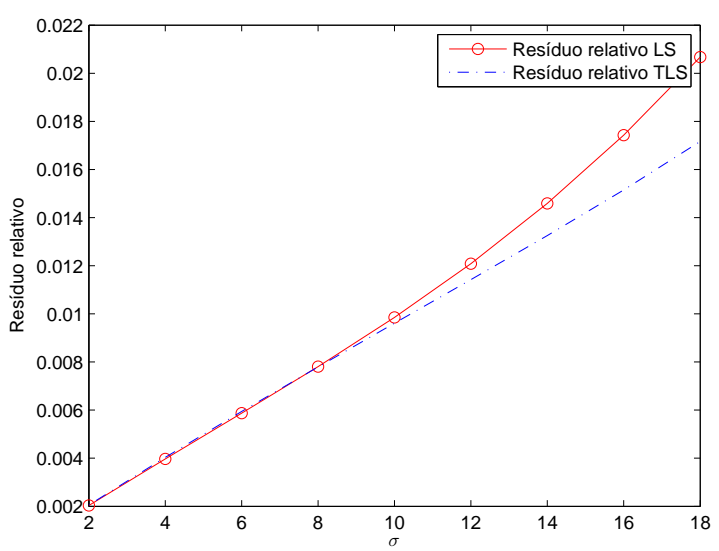

Figura 3: Resíduo relativo

Vemos que os erros relativos associados as soluções LS e TLS são muito próximos. No caso do resíduo relativo, vemos que para valores grandes de $\sigma$ a solução TLS é melhor.

As estimativas dos valores dos parâmetros $\alpha$ e $\omega$ são apresentados na tabela 3 e graficamente nas figuras 4 e 5 .

Tabela 3: Erros Relativos

\begin{tabular}{ccccc}
\hline$\sigma$ & $\left\|\widetilde{\alpha}_{L S}-\alpha\right\| /\|\alpha\|$ & $\left\|\widetilde{\alpha}_{T L S}-\alpha\right\| /\|\alpha\|$ & $\left\|\widetilde{\omega}_{L S}-\omega\right\| /\|\omega\|$ & $\left\|\widetilde{\omega}_{T L S}-\omega\right\| /\|\omega\|$ \\
\hline 2 & 0.0057111 & 0.0061896 & 0.0003957 & 0.0004067 \\
4 & 0.0101561 & 0.0117233 & 0.0007376 & 0.0007801 \\
6 & 0.0140621 & 0.0166266 & 0.0010293 & 0.0011192 \\
8 & 0.0184575 & 0.0209818 & 0.0012792 & 0.0014233 \\
10 & 0.0244831 & 0.0249581 & 0.0015022 & 0.0016927 \\
12 & 0.0330535 & 0.0288461 & 0.0017201 & 0.0019288 \\
14 & 0.0447341 & 0.0330901 & 0.0019607 & 0.0021337 \\
16 & 0.0598983 & 0.0383309 & 0.0022535 & 0.0023098 \\
18 & 0.0790414 & 0.0456766 & 0.0026299 & 0.0024639 \\
\hline
\end{tabular}




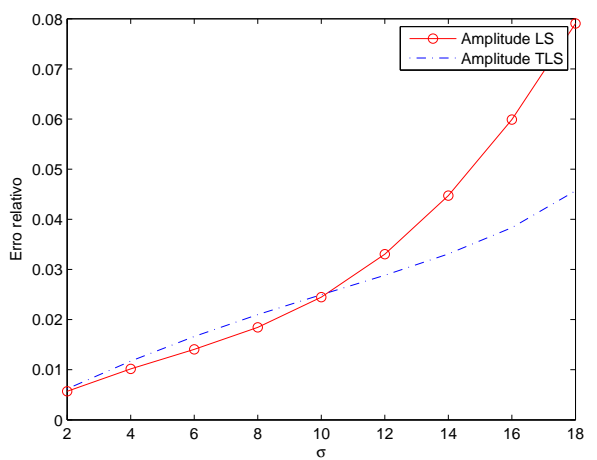

Figura 4: Amplitude

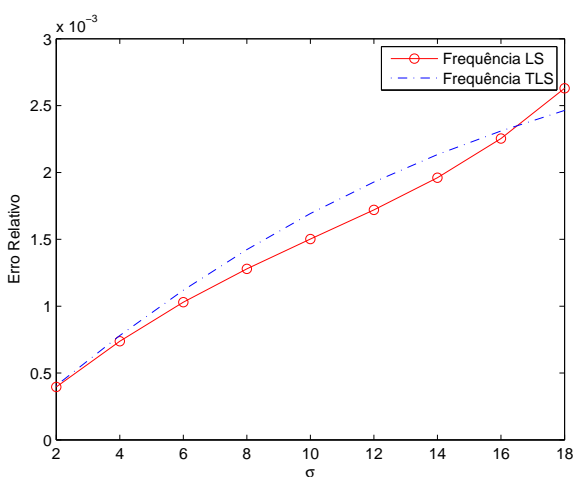

Figura 5: Frequência

Vemos que ambos os métodos fornecem boas estimativas das frequências $\omega$ independente do valor de $\sigma$. A mesma observação vale para os parâmetros $\alpha$ quando $\sigma$ é pequeno. Porém, para valores maiores de $\sigma$, vemos que o método TLS proporciona uma melhor precisão, confirmando que o método TLS é uma excelente alternativa para problemas onde o ruído afeta a matriz e o vetor de dados.

\section{Referências}

[1] A. Doicu, T. Trautmann, and F. Schreier, "Numerical Reguralization for Atmospheric Inverse Problems", Springer-Verlag Berlin Heidelberg, pp. 254-256, 2010.

[2] F. S. Bazán V., CGLS-GCV: a hybrid algorithm for low-rank-deficient problems, Applied Numerical Mathematics, Vol. 47, pp. 91-108, 2003.

[3] G. H. Golub, and C. F. Van Loan, An analysis of the total least square problem, SIAM J. Number. 6, Anal., Vol. 17, pp. 883-893, 1980.

[4] G. H. Golub, AND C. F. Van Loan, "Matriz Computations". 3.ed Maryland: Jhon Hopkins University Press, 1996.

[5] P. C. Hansen, "Rank-deficient and discrete ill-possed problems", Philadelphia: SIAM, 1998.

[6] P. C. Hansen, "Discrete inverse problems: Insight and algorithm", Philadelphia: SIAM, 2010.

[7] S. Van Huffel. and J. Vandewalle, "The Total Least Square Problems - Computational Aspects and Analysis", SIAM, Philadelphia, PA, 1991. 